\title{
Sur les chemins de l'écriture avec François Bon
}

\section{On the paths of writing with François Bon}

\author{
Zeinab GOLESTANI DERO' 1 (1)
}

Phd student of French literature, Shahid Beheshti University, French Department, Tehran, Iran

ORCID: Z.G.D. 0000-0002-3659-5347

Corresponding author: Zeinab GOLESTANI DERO, Shahid Beheshti University, French Department, Evin Street, Velenjak, Tehran, Iran

E-mail: z_golestanidero@sbu.ac.ir

Ecrivain contemporain français, François Bon est né en 1953 en Vendée à l'est de la France. Après avoir poursuit ses études en ingénieur mécanique, il a publié à 29 ans, en s'inspirant du milieu où il a grandi, son premier roman intitulé La Sortie d'Usine. Désormais et après avoir fait de 1980 à 1982 des études en philosophie, cet ingénieur s'est consacré entièrement à la littérature et s'est occupé de l'organisation des ateliers de l'écriture créative auprès des gens en difficulté sociale. Son vaste corpus comprend plus de trente livres dans divers domaines de l'écriture y compris roman, récit, poème en prose et roman pour adolescent. Or, il n'a jamais oublié d'établir des liens et des dialogues avec des musiciens, des peintres, des photographes, des philosophes, des sociologues et des urbanistes.

Portant un regard attentif au monde qui l'environne, l'œuvre de Bon explore la vie de tous les jours des gens actuels et ordinaires subissant des règles non écrites du monde urbain actuel. C'est en s'appuyant sur ce point de vue qu'elle relève du réel, ce 
qui ne s'appuie pas sur un réalisme dur et pur, mais sur le contact avec le monde. C'est ce réel que Bon cherche à produire et non pas reproduire et qui, en révélant l'autre face d'une culture et d'une époque, introduit dans l'œuvre de Bon des fragments de la vie des autres et des bribes de soi. Effectivement, pour lui, l'écriture est écrire de soi, écrire non pas sur le monde, mais avec le monde, avec la littérature, avec les mots, et avec toutes les voix qui murmurent dans le monde qui entoure l'écrivain.

Son attachement au monde contemporain et sa tentation pour le découvrir et redécouvrir apparaît aussi dans sa faveur pour le monde virtuel de l'Internet. Son site remue.net qui est mis en ligne en 2001 propose des études et des critiques bien intéressantes sur la production littéraire contemporaine. En plus, il a créé en 2005 son site personnel tierslivre.net qui offre la possibilité de trouver ses œuvres, ses débats et ses prises de positions. Il est aussi le fondateur de l'une des premières maisons d'édition numérique, à savoir publie.net qui contient une riche sélection de l'écriture contemporaine.

Le présent propos est le résultat d'un dialogue avec cet écrivain ingénieur qui est fait en ligne et qui comprend ses idées et opinions sur l'écriture et notamment l'écriture urbaine.

\section{Quand et comment vous avez commencé votre carrière d'écrivain ?}

Je n'ai jamais considéré écrire comme une carrière, sinon il aurait mieux valu que je reste dans mon premier métier. C'est une liberté d'être indépendant, de mener son travail artistique sans rien demander à personne, c'est assez difficile comme ça. Il faudrait aussi examiner comment le mot écrivain, venu tardivement (au XVIIe siècle pour la France) n'est peut-être pas le mieux approprié pour décrire ce que la mutation numérique transforme aussi à l'auteur. J'ai publié mon premier livre à vingt-neuf ans, j'ai eu la chance d'une relation primordiale à la lecture dès l'enfance, après c'est beaucoup de hasards et de traverses.

Parmi les écrivains classiques, vous préférez lesquels ? Est-ce que vous vous attachez au courant du réalisme et certainement à Balzac ? Que pensez-vous à propos de son œuvre?

Une fois qu'on a publié un premier livre, on peut prendre un moment pour chercher par quel chemin arbitraire de hasard on a disposé d'un minimum de lectures fondatrices. 
Mais ensuite il faut embrasser la littérature comme un grand corps, comprendre ses ramifications, ses arborescences. Il ne s'agit plus de " préférer » un tel ou une telle. Balzac a évidemment eu une grande importance dans mon cas, parce que j'ai eu la chance de m'y immerger dès l'adolescence. Un monde d'illusion qui remplaçait le monde ordinaire. Mais tout son travail naît de ses nouvelles fantastiques, ou de choses carrément magiques, comme La peau de chagrin venue de Perse, ou le mysticisme de Louis Lambert. On n'a pas vraiment besoin du mot réalisme pour écrire.

\section{Mais qu'est-ce qui est nécessaire pour écrire? Connaître la littérature littéraire? Voire autrement le monde?}

Pour écrire, ce qui est nécessaire c'est du papier et un crayon, ou un téléphone, ou du sable. Il n'y a pas d'autorisation à demander, pour écrire.

En tant qu'étudiante en littérature, je suis obligée de lire depuis huit ans des théories littéraires, mais je me demandais toujours pourquoi ni moi ni aucune de mes camarades ni mes professeurs n'ont jamais pu écrire, ou avoir une production littéraire.

C'est vraiment dommage. Dans de très nombreux pays désormais, et pas seulement le Canada ou les USA, l'enseignement de la création littéraire est parfaitement reconnu. Mais ce n'est pas la fonction des études littéraires de préparer au métier d'écrivain. Dans la tradition française, il y a une extraordinaire diversité des chemins qui ont conduit à l'écriture nos auteurs et autrices, et pas seulement des professions savantes comme médecin ou ingénieur. De très nombreux auteurs (je pense à Artaud, à Koltès, à Michaux) n'ont jamais fait d'études universitaires.

\section{C'est la base philosophique des études scolaires en France qui pousse les jeunes} à réfléchir sur le monde et à écrire ce qu'ils pensent? Autrement dit, quelles matières scolaires peuvent ouvrir ces chemins vers l'écriture?

Je me suis beaucoup battu, depuis près de 20 ans, avec beaucoup d'autres auteurs et enseignants, pour que l'école - à tous les âges - s'ouvre à des pratiques de création littéraire, ou même disons à l'écriture comme pratique, ou écriture d'invention comme on a pu la nommer. En France, longtemps, avant qu'un enfant puisse apprendre à toucher un instrument de musique, il devait apprendre deux ans le solfège, puis faire 
un an de flûte à bec : en littérature on en est encore là. L'écriture créative commence lentement à être reconnue, il y a des masters de création littéraire à l'université, mais globalement ce n'est pas un combat qu'on a gagné. Après, il n'y a besoin de l'autorisation de personne pour se lancer dans l'écriture. C'est par la lecture des auteurs qui nous ont précédé, qu'on apprend, ce n'est pas la tâche de l'école d'y procéder.

Et aussi, le Château de Kafka, et ses œuvres, on les trouve dans vos œuvres; pourriez-vous donner quelques explications à propos de ce choix ?

J'ai eu la chance aussi de lire Kafka, quasiment en entier sauf son Journal, découvert plus tard, vers mes dix-sept ans. Après je n'ai quasiment pas lu jusqu'à mes vingt-six ans, quand l'écriture a pris la première place, donc il est toujours resté très central, affectivement aussi, comme Proust, Gracq, Michaux, Sarraute et quelques autres vieux compagnons, ou Rabelais, ou Saint-Simon.

\section{Comment définissez-vous l'écriture ? Quelle est le but de l'écriture ?}

On s'interroge sur la relation du langage aux choses, on cherche dans le langage ce qui le relie à ce qui obscurément nous entoure. On n'a pas besoin de plus.

Et c'est dans ce contact avec le monde que naît l'écriture? II nous faut donc prêter l'oreille au murmure de tout ce qui nous entoure?

C'est une très belle, formule, comme vous I'utilisez ! Et oui, effectivement, tout ce qui touche à l'ouïe est l'univers le plus sensible pour l'écriture.

J'ai entendu à propos de Gide, à chaque fois qu'il dirigeait un cours d'écriture, il demandait aux participants de traduire d'abord une grande œuvre classique. Et vous avez des traductions aussi. Comment cette traduction influence l'écriture?

Traduire c'est écrire. C'est un laboratoire d'écriture qu'on se donne parce qu'on a des choses de son propre travail à démêler, qui ne sont pas mûres encore. Cela permet d'avancer un chantier en profondeur, sans que cela procède de ses contenus personnels. J'ai toujours bricolé des bribes de traduction, longtemps avant les expériences récentes qui vont jusqu'à des livres. Pour Gide je ne sais pas, je ne l'ai jamais lu vraiment. 


\section{Alors la traduction donne-t-elle naissance à une nouvelle œuvre littéraire?}

On peut répondre positivement dans de nombreux exemples, le plus célèbre étant comment la réinvention des récits d'Edgar Poe par Baudelaire lui a permis de formuler et engendrer son propre travail. Le travail d'André Markowicz dans ce domaine (ou Henri Meschonnic, Yves Bonnefoy et tant d'autres) en parlent très bien.

Vous dirigiez des ateliers intitulés Écrire la ville, comment écrire la ville ? Fautil chercher des images au sein de la ville ? Comment reconstruire des images de la ville ? Cette reconstruction des images, est-elle différente de la description ?

La réflexion sur les ateliers d'écriture a commencé pour moi vers 1993, sans discontinuer depuis. On travaille les structures du récit, la poétique du langage, l'intuition ou le rêve, les dialogues, et bien sûr aussi ce qui nous entoure. La question urbaine, depuis plusieurs décennies, est primordiale pour comprendre les formes sociales, les territoires, ce qui change des structures familiales ou sociales. La ville est un enjeu esthétique récent, quand Walter Benjamin met en évidence la rupture qu'elle représente pour Baudelaire. Il semble que désormais, en France tout au moins, le modèle urbain se soit globalisé ou, via la façon dont le numérique irrigue la totalité de notre relation au dehors, on puisse peut-être ouvrir d'autre chantiers.

Cette écriture de la ville est à la recherche de quoi ? Cherche-t-elle à faire habitable la ville ? Cherche-t-elle les coins oubliés de la ville ? Cette écriture, estelle pour vous un moyen de sauver ces angles invisibles de l'oubli ?

La littérature ne cherche pas à transformer le monde, ni à sauver quoi que ce soit. Il y a des gens très bien qui le font (je pense à l'École urbaine de Lyon) et nous, ce que ces modes de socialité changent au récit nous suffit.

Pourquoi alors vous choisissez d'écrire sur les ouvrières de Daewoo ou bien sur les cités HLM. Pourquoi illustrer la vie des gens qui « vivent dans une dent gâtée ॥ ? ou bien il s'agit de I'histoire de toute l'humanité?

A l'origine, Daewoo était une commande du centre dramatique de Nancy, alors que je collaborais depuis plusieurs années avec le metteur en scène qui le dirigeait, Charles Tordjman. Un fait divers qui secouait en profondeur cette vieille région industrielle. 
Pour écrire les monologues et dialogues de théâtre, j'ai été amené à me rendre plusieurs fois sur les lieux, et construire des sortes de vies fictives à mes personnages, c'est ainsi qu'est né le livre.

Comment faire entendre les voix de la ville dans le roman ? Dans Sortie d'Usine, en lisant le texte, on sent être au milieu des instruments industriels, ou dans Paysage fer, c'est la voie ferrée qui se fait entendre, la lecture suit les mêmes mouvements que le train. Il s'agit d'une autre manière de reconstruire la ville ?

J'ai eu la chance, enfant, d'être au milieu de deux univers, les livres côté maternel, avec le grand-père et ma propre mère instituteur et institutrice, et la mécanique et les machines côté paternel - nous vivions dans un garage. Pour Sortie d'usine comme pour Paysage Fer, c'est cet environnement de proximité qui a déterminé la forme du livre.

C'est tout à fait intéressant pour moi, parce qu'en lisant Paysage fer, je retournais de temps en temps à Sortie d'Usine et je sentais les mêmes choses.

Il y a pourtant vingt ans d'écart entre les deux. Cela renvoie à cette expression de William Faulkner, disant qu'on a chacun un petit timbre-poste à explorer.

L'écriture, ouvre-t-elle une voie pour faire des échanges, des communications au sein des villes qui ne permettent pas aux gens de communiquer?

Communiquer n'est pas l'affaire de l'écriture.

Comment regarder les espaces urbains quand on veut écrire la ville?

La question de voir n'est pas limitée à la ville. Apprendre, progresser dans l'écriture, c'est passer par les peintres, les architectes, la musique, les voyages aussi. Alors on a peut-être une chance de voir un peu mieux. J'essaye d'être très attentif aux artistes d'autres champs disciplinaires.

Et je pense que comme Michel Butor sur l'œuvre duquel j'ai travaillé quand j'étais étudiante en master, vous avez aussi des collaborations avec des artistes? 
Bien sûr, c'est essentiel. Peintres, photographes, musiciens, architectes, on se forme énormément à s'appuyer sur les logiques d'autres disciplines. II faut aussi y inclure les scientifiques, et quelques philosophes. Chacun se fait son cercle, son territoire, mais souvent cela prend plus d'importance que la fréquentation d'autres écrivains...

Et l'industrie, est-ce qu'on peut s'en passer quand on veut écrire la ville ?

Qui est « on » ? Proust parle de duchesses et de concierges, parce que c'était son monde. Je considère comme une grande chance d'avoir pu pénétrer un peu le monde de l'industrie, absurdement protégé souvent. Je continue de le faire autant que je peux.

Ce qui m'attire dans ce regard vers l'industrie, c'est qu'en lisant votre œuvre, je sens que les personnages acceptent cette mode de vie, cette industrie qui est aujourd'hui une partie importante de notre vie quotidienne, et cela malgré ceux qui cherche à s'en fuir, à chercher un abri ou pour bien dire, un pays perdu dans le passé, dans tout ce qui n'existe peut-être aujourd'hui que dans les livres d'histoire. Je l'appelle vivre dans la géographie (qui contient elle-même des couches historiques).

La notion de géographie est très importante, c'est ce que je dois aussi à ma lecture de Julien Gracq. Je l'assume toujours dans ses récentes évolutions, concernant le développement durable et l'écologie, l'évolution urbaine.

La politique, est-elle indissociable de l'écriture ? Certains critiques regardent vos œuvres comme des œuvres politiques, est-ce que vous en êtes d'accord ?

Ça ne me concerne pas. On a assez de travail avec l'écriture. Les livres qui se mêlent de prétention politique ont en général peu d'avenir, sinon Bossuet ou Hugo. La poésie a une autre ambition.

En lisant vos œuvres, on a le sentiment que ce qui, à la fin, sauve des gens ordinaires et marginalisés de l'oubli, c'est l'art et l'écriture, je veux dire que c'est une production littéraire ou artistique, soit un journal intime, soit un tableau de peinture, etc., qui ouvre la voie de salut ; votre écriture, cherche-t-elle aussi ce but? 
Pas d'autre but que se connaître un peu mieux soi-même. On n'est pas en charge de sauver qui que ce soit.

\section{Comment on peut se connaître à travers l'écriture?}

Si on avait la réponse, on n'aurait pas besoin de continuer ! c'est un chemin que bien d'autres accomplissent aussi. Le travail du rêve, par exemple...

\section{Comment faut-il définir un roman ? On dit, de votre part, que vous n'écrivez pas des romans ; comment peut-on appeler cette écriture?}

On peut appeler ça écrire, tout simplement. Et encore, on est tous aujourd'hui immergés dans des usages où la photographie, le film, la voix aussi permettent d'élargir le champ, ou de donner plus d'intensité, à notre nécessité de langage. Le concept de publication, y compris sur le web, ou via une chaîne vidéo, produit désormais des formes aussi puissantes qu'a pu l'être le livre, dans sa longue histoire. La notion de genre (roman, poésie, théâtre, etc) est tardive et obsolète : elle structure toujours I'université française, de même qu'elle divise absurdement l'écriture en siècles, mais ça concerne uniquement l'université et sa sclérose. L'interrogation dont on parlait au début, le langage confronté à l'inconnu du présent, ça suffit largement, il faut apprendre intérieurement à se libérer des formes héritées, puis avalées par l'industrie culturelle - dans notre petit monde occidental en tout cas. 